\title{
MicroRNA-497-5p inhibits proliferation and invasion of non-small cell lung cancer by regulating FGF2
}

\author{
XIAORI HUANG, LEI WANG, WEI LIU and FEI LI \\ Department of Respiratory Medicine, People's Hospital of Rizhao, Rizhao, Shandong 276826, P.R. China
}

Received July 8, 2018; Accepted January 16, 2019

DOI: $10.3892 / 01.2019 .9954$

\begin{abstract}
Increasing number of microRNAs (miRNAs) have been reported to play an important role in the development and progression of non-small cell lung cancer (NSCLC). In particular, microRNA-497-5p (miR-497-5p) has been proposed as a tumor suppressor miRNA in human cancers. However, the role of miR-497-5p and its potential molecular mechanism associated with NSCLC are less studied. Therefore, the role of miR-497-5p in the pathogenesis of NSCLC was investigated. In the present study, the expression of miR-497-5p was significantly downregulated in NSCLC. Moreover, overexpression of miR-497-5p inhibited the proliferation and invasion of NSCLC cells by suppressing FGF2. In addition, FGF2 was a downstream target of miR-497-5p in NSCLC. FGF2 was upregulated in NSCLC promoting cell proliferation and invasion. Overexpression of FGF2 impaired the inhibitory effect of miR-497-5p in NSCLC. Taken together, these results demonstrate that miR-497-5p is a tumor suppressor miRNA and demonstrate its potential for future use in the treatment of human NSCLC.
\end{abstract}

\section{Introduction}

Lung cancer is one of the most common malignant tumors in the world and has become a major cause of high mortality in China's urban population (1). Primary lung cancer includes small cell lung carcinoma (SCLC) and non-SCLC (NSCLC), and NSCLC accounts for $\sim 85 \%$ of all lung cancer cases (2). Although treatments have improved significantly in recent years, the prognosis of patients with NSCLC remains poor, with a 5-year survival rate of only $\sim 16 \%$ (3). Survival rates have been found to be closely associated with the lung cancer stage. Patients with stage I-III NSCLC can be treated surgically with a 5-year survival rate of $25-80 \%$ (4). Stage IV patients with NSCLC are often treated by chemotherapy to

Correspondence to: Dr Xiaori Huang, Department of Respiratory Medicine, People's Hospital of Rizhao, 126 Tai'an Road, Rizhao, Shandong 276826, P.R. China

E-mail:vjo320615@163.com

Key words: miR-497-5p, proliferation, invasion, FGF2, non-small cell lung cancer relieve symptoms. Even so, patients with stage IV NSCLC have a poor prognosis with a 5-year survival rate of only $2 \%$ (5). Unfortunately, NSCLC is often diagnosed in advanced stage. Therefore, finding effective screening strategies for early diagnosis is necessary. It would likely significantly reduce the morbidity and mortality of NSCLC.

It has been reported that microRNAs (miRNAs) inhibit expression of target genes by disrupting genes or impeding translation of mRNA (6). Especially, increased microRNAs have been identified as lung cancer biomarkers (7). For example, miR-215 inhibited the progression of NSCLC through targeting MMP-16 (8). Wei and Ran (9) reported that miR-20a promoted proliferation and invasion by directly targeting EGR2 in NSCLC. Recently, the specific function of miR-497 has attracted our attention. It was proven that miR-497 can be used as an effective biomarker to diagnose and predict prognosis of osteosarcoma (10). Abnormal expression and function of miR-497 have been identified in various types of human of cancer. For instance, miR-497 was downregulated and suppressed the development of renal cell carcinoma through targeting VEGFR-2 (11). Wu et al (12) proposed that miR-497 inhibited proliferation and induced apoptosis via the Bcl-2/ Bax-caspase-9-caspase-3 pathway in HUVECs. It was also reported that miR-497 accelerated apoptosis of cervical cancer cells through negatively regulating the MAPK/ERK signaling pathway (13). However, it has been reported that miR-497 can also express high and enhanced metastasis by inhibiting SMAD7 in oral squamous cell carcinoma (14). These results led us to further explore the role of miR-497-5p in NSCLC.

As a member of FGF family, fibroblast growth factor 2 (FGF2) is involved in mitogenic and proliferative effect (15). FGF2 was reported to be upregulated in breast cancer and mediated cell migration and invasion (16). Moreover, FGF2 was found to facilitate cell invasion in pancreatic cancer (17). Joy et al (18) demonstrated that FGF2 could promote cell proliferation in human astrocytes and glioma. As a biomarker, FGF2 can predict the prognosis of patients with glioma (19). In addition, the interaction between miRNAs and FGF2 has been reported in human cancers, such as miR-15-16 (20). However, the regulatory mechanism between miR-497-5p and FGF2 is unclear and an in-depth study is desired urgently in NSCLC. In the present study, the expression of miR-497-5p and its regulatory mechanism in NSCLC were investigated. Moreover, the relationship between miR-497-5p and FGF2 was also explored in NSCLC. 


\section{Materials and methods}

Clinical tissues. This experiment was approved by the Institutional Ethics Committee of People's Hospital of Rizhao (Rizhao, China) (Approval no. 2017-5, February 15, 2017). Informed consent was signed by all patients. In People's Hospital of Rizhao, 108 NSCLC tissues and adjacent normal tissues were obtained from patients. None of the patients with NSCLC received treatment before surgery. Tissues were then frozen in liquid nitrogen and stored in a $-80^{\circ} \mathrm{C}$ refrigerator for further experiments.

Cell culture. Human H1299, A549 and SPC-A1 cell lines and non-tumorigenic bronchial epithelium cell line BEAS-2B (Homo sapiens) were obtained from the American Type Culture Collection (ATCC; Manassas, VA, USA). These cells were then incubated with $10 \%$ fetal bovine serum (FBS) in Dulbecco's modified Eagle's medium (DMEM; Thermo Fisher Scientific, Inc., Waltham, MA, USA). They were placed in an incubator with $5 \% \mathrm{CO}_{2}$ at $37^{\circ} \mathrm{C}$.

$R T-q P C R$. Total RNA containing miRNA was extracted using TRIzol reagent (Invitrogen; Thermo Fisher Scientific, Inc.) to quantify the expression of miR-497-5p in NSCLC. Reverse transcription quantitative polymerase chain reaction (RT-qPCR) was performed on ABI 7500 Fast Real-Time PCR system (Applied Biosystems; Thermo Fisher Scientifc, Inc.) using SYBR-Green PCR Master Mix (Thermo Fisher Scientifc, Inc.). The primer sequences used for $\mathrm{qPCR}$ were: miR-497-5p 5'-CCTTCAGCAGCACACTGTGG-3' (forward) and 5'-CAGTGCAGGGTCCGAGGTAT-3' (reverse); U6 5'-CTCGCTTCGGCAGCACA-3' (forward) and 5'-AACGCTTCACGAATTTGCGT-3' (reverse); FGF2 5'-ACTGGCTTCTAAATGTGTTACG-3' (forward) and 5'-TTGGATCCAAGTTTATACTGCC-3' (reverse); GAPDH 5'-TGGTATCGTGGAAGGACTCA-3' (forward) and 5'-CCAGTAGAGGCAGGGATGAT-3' (reverse). U6 and GAPDH were used as controls. Their expression was analyzed according to the $2^{-\Delta \Delta c q}$ method (21).

Cell transfection. miR-497-5p mimic and inhibitor were used to mimic and inhibit miR-497-5p expression. FGF2 siRNA was used to inhibit FGF2 expression. miR-497-5p mimic and inhibitor, and FGF2 siRNA (si-FGF2) were obtained from Shanghai GenePharma Co., Ltd. (Shanghai, China). They were then transferred to A549 cells with Lipofectamine 2000 (Invitrogen; Thermo Fisher Scientific, Inc.) based on the manufacturer's protocols.

Cell proliferation. Cell proliferation was measured by the CCK-8 assay based on the manufacturer's instructions. First, $4 \times 10^{4}$ cells were plated and incubated in 96-well plates for 0 , 24,48 and $72 \mathrm{~h}$. They were placed in an incubator with $5 \% \mathrm{CO}_{2}$ at $37^{\circ} \mathrm{C}$. Next, $10 \mu \mathrm{l}$ of CCK-8 reagent was added to each well for $2 \mathrm{~h}$ (Dojindo Laboratories, Kumamoto, Japan). Finally, they were detected using a microplate reader (Molecular Devices LLC, Sunnyvale, CA, USA) at an absorbance of $450 \mathrm{~nm}$.

Cell invasion. Cell invasion ability was assessed by Transwell assay. First, $5 \times 10^{4}$ A549 cells in serum-free medium were seeded in a Matrigel-coated Transwell chambers $(8-\mu \mathrm{m}$ pore size; BD Biosciences, San Jose, CA, USA). A549 cells were then placed in the upper chamber and the lower chamber was filled with $10 \% \mathrm{FBS}$. The cells were then cultured at $37^{\circ} \mathrm{C}$ for $48 \mathrm{~h}$ to measure cell invasion. The cells were stained with $0.1 \%$ crystal violet. A light microscope (Olympus Corporation, Tokyo, Japan) was used to count invading cells.

Dual-Luciferase report assay. The 3'-UTR of wild or mutant type FGF2 was inserted into the pmirGLO plasmids (Shanghai GenePharma Co., Ltd.) for luciferase reporter assay. Then, the 3'-UTR of wild or mutant type FGF2 and miR-497-5p mimic were transfected into A549 cells. Luciferase activity was observed by a Dual-Luciferase assay system (Promega, Madison, WI, USA).

Western blotting. RIPA lysis buffer was used to extract the protein samples. Proteins were then separated through a $10 \%$ SDS-PAGE and incubated with 5\% skim milk in polyvinylidene difluoride (PVDF) membranes at room temperature. Next we incubated the membranes with anti-FGF2 (dilution 1:1,000; rabbit polyclonal; cat. no. 208687; Abcam, Cambridge, MA, USA), anti-GAPDH (dilution 1:1,000; rabbit monoclonal; cat. no. ab9485; Abcam) antibodies at $4^{\circ} \mathrm{C}$ overnight, followed by incubation with goat polyclonal anti-rabbit IgG secondary antibodies (dilution 1:2,000; cat. no. ab6721; Abcam). Protein expression levels were then measured by ECL (Pierce; Thermo Fisher Scientific, Inc.).

Statistical analysis. Data were analyzed using SPSS 19.0 (IBM Corp., Armonk, NY, USA) and GraphPad Prism 6 (GraphPad Software, Inc., La Jolla, CA, USA). Data are presented as mean \pm standard deviation (SD). Differences were calculated according to one-way analysis of variance (ANOVA) with the post hoc Tukey's test or Student's t-test. The correlation between the miR-497-5p and FGF2 was analyzed through Spearman's two-tailed test. The association between the clinicopathological features of NSCLC and miR-497-5p or FGF2 was calculated by the Chi-squared test $\left(\chi^{2}\right)$. The overall survival was analyzed by the Kaplan-Meier method and the $\log$ rank test. Significant differences were defined as $\mathrm{P}<0.05$.

\section{Results}

Downregulation of miR-497-5p identified in NSCLC tissues and cell lines. First, mRNA expression of miR-497-5p was observed in NSCLC tissues. Downregulation of miR-497-5p was detected in NSCLC tissues compared to adjacent normal tissues (Fig. 1A). In the NSCLC cell lines, downregulation of miR-497-5p was also identified. The expression levels of miR-497-5p were significantly decreased in the H1299, A549 and SPC-A1 cell lines compared to BEAS-2B cells (Fig. 1B). In addition, the relationship between miR-497-5p abnormal expression and clinicopathological features of NSCLC patients was analyzed. Based on the expression of miR497-5p, these cases were divided into a high miR-497-5p expression group and a low expression group, based on the median value of miR-497-5p expression levels in NSCLC patients as a cut-off point. We found that low miR-497-5p expression was associated with TNM stage $(\mathrm{P}=0.018)$ and 
A

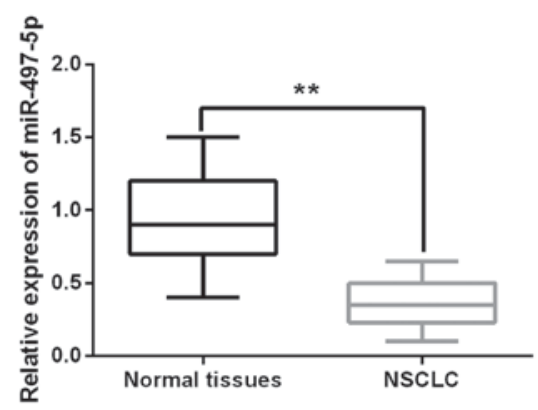

B

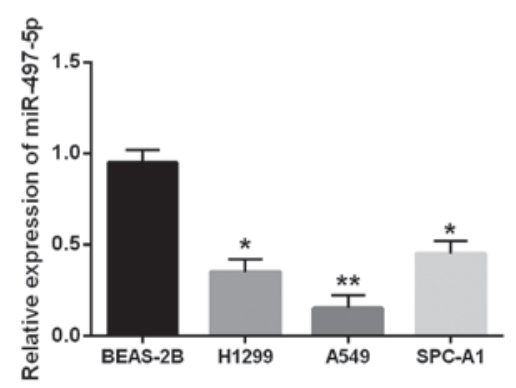

C

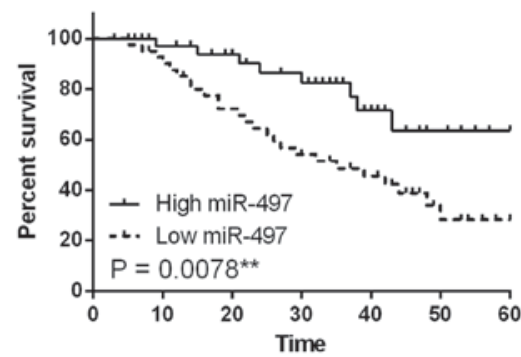

Figure 1. Downregulation of miR-497-5p was identified in NSCLC. (A) The expression of miR-497-5p in NSCLC tissues detected via qRT-PCR. (B) The miR-497-5p expression in H1299, A549, SPC-A1 and BEAS-2B cell lines. (C) Low miR-497-5p expression was correlated with shorter overall survival of NSCLC patients. ${ }^{*} \mathrm{P}<0.05,{ }^{* *} \mathrm{P}<0.01$.

distant metastases ( $\mathrm{P}=0.02$; Table I). Besides, low miR-497-5p expression predicted a poor prognosis, with NSCLC patients having a shorter overall survival ( $\mathrm{P}=0.0078$; Fig. 1C). Based on these results, we suspected that miR-497-5p was involved in the tumorigenesis of NSCLC.

Overexpression of miR-497-5p inhibited cell proliferation and invasion in NSCLC. Next, miR-497-5p mimics or inhibitor was transfected into A549 cells to explore its role in NSCLC. The miR-497-5p mimics were found to enhance miR-497-5p expression (Fig. 2A) and miR-497-5p inhibitor reduced its expression (Fig. 2B). The ability of cell proliferation was then measured in transfected A549 cells. The CCK-8 assay indicated that upregulation of miR-497-5p suppressed proliferation of A549 cells (Fig. 2C). In contrast, knockdown of miR-497-5p promoted cell proliferation in NSCLC cells (Fig. 2D). In addition, the same effect of miR-497-5p was identified for cell invasion in NSCLC. miR-497-5p mimics significantly impaired cell invasive ability, while miR-497-5p inhibitor promoted invasion of A549 cells (Fig. 2E). Therefore, overexpression of miR-497-5p was found to suppress cell proliferation and invasion in NSCLC.
Table I. Relationship between miR-497-5p expression and the clinicopathological characteristics of NSCLC patients.

\begin{tabular}{|c|c|c|c|c|}
\hline \multirow[b]{2}{*}{ Characteristics } & \multirow{2}{*}{$\begin{array}{l}\text { No. of cases } \\
\quad(n=108)\end{array}$} & \multicolumn{2}{|c|}{ miR-497-5p } & \multirow[b]{2}{*}{ P-value } \\
\hline & & High & Low & \\
\hline Age (years) & & & & 0.12 \\
\hline$\geq 60$ & 40 & 12 & 28 & \\
\hline$<60$ & 68 & 28 & 40 & \\
\hline Sex & & & & 0.28 \\
\hline Male & 48 & 20 & 28 & \\
\hline Female & 60 & 22 & 38 & \\
\hline Tumor size (cm) & & & & 0.56 \\
\hline$<5$ & 46 & 21 & 25 & \\
\hline$\geq 5$ & 62 & 22 & 40 & \\
\hline TNM stage & & & & $0.018^{\mathrm{a}}$ \\
\hline $\mathrm{I}+\mathrm{II}$ & 33 & 11 & 22 & \\
\hline III + IV & 75 & 24 & 51 & \\
\hline Distant metastases & & & & $0.02^{\mathrm{a}}$ \\
\hline Negative & 72 & 27 & 45 & \\
\hline Positive & 36 & 13 & 23 & \\
\hline Differentiation & & & & 0.35 \\
\hline $\mathrm{I}+\mathrm{II}$ & 58 & 20 & 28 & \\
\hline III + IV & 50 & 20 & 30 & \\
\hline
\end{tabular}

Statistical analyses were performed by the $\chi^{2}$ test. ${ }^{\text {a }}<<0.05$ was considered significant.

FGF2 is a downstream target of miR-497-5p in NSCLC. TargetScan (http://www.targetscan.org) was used to search for potential binding sites of the theoretical target gene with miR-497-5p. Among the predicted targets, the FGF2 gene was selected for further analysis because of its vital role in promoting tumor progression. The prediction of TargetScan showed that miR-497-5p had a binding site to the 3'UTR of FGF2 (Fig. 3A). Luciferase assay was then performed to confirm the above prediction. The results showed that the miR-497-5p mimics significantly reduced the luciferase activity of wild-type FGF2. However, the luciferase activity of the mutant type FGF2 was not affected by the miR-497-5p mimics (Fig. 3B). In addition, a negative correlation between miR-497-5p and FGF2 expression was identified in NSCLC tissues $\left(\mathrm{P}<0.0001, \mathrm{R}^{2}=0.5738\right.$, Fig. $\left.3 \mathrm{C}\right)$. To further confirm the relationship between them, the expression of FGF2 was detected in A549 cells with miR-497-5p mimics or inhibitor. The results indicated that miR-497-5p mimics obviously suppressed FGF2 expression, while the miR-497-5p inhibitor enhanced the expression level of FGF2 (Fig. 3D and E). FGF2 is a direct target of miR-497-5p, and miR-497-5p negatively regulated FGF2 expression in NSCLC.

Upregulation of FGF2 promotes cell proliferation and invasion in NSCLC. Then, FGF2 was identified to be upregulated in 

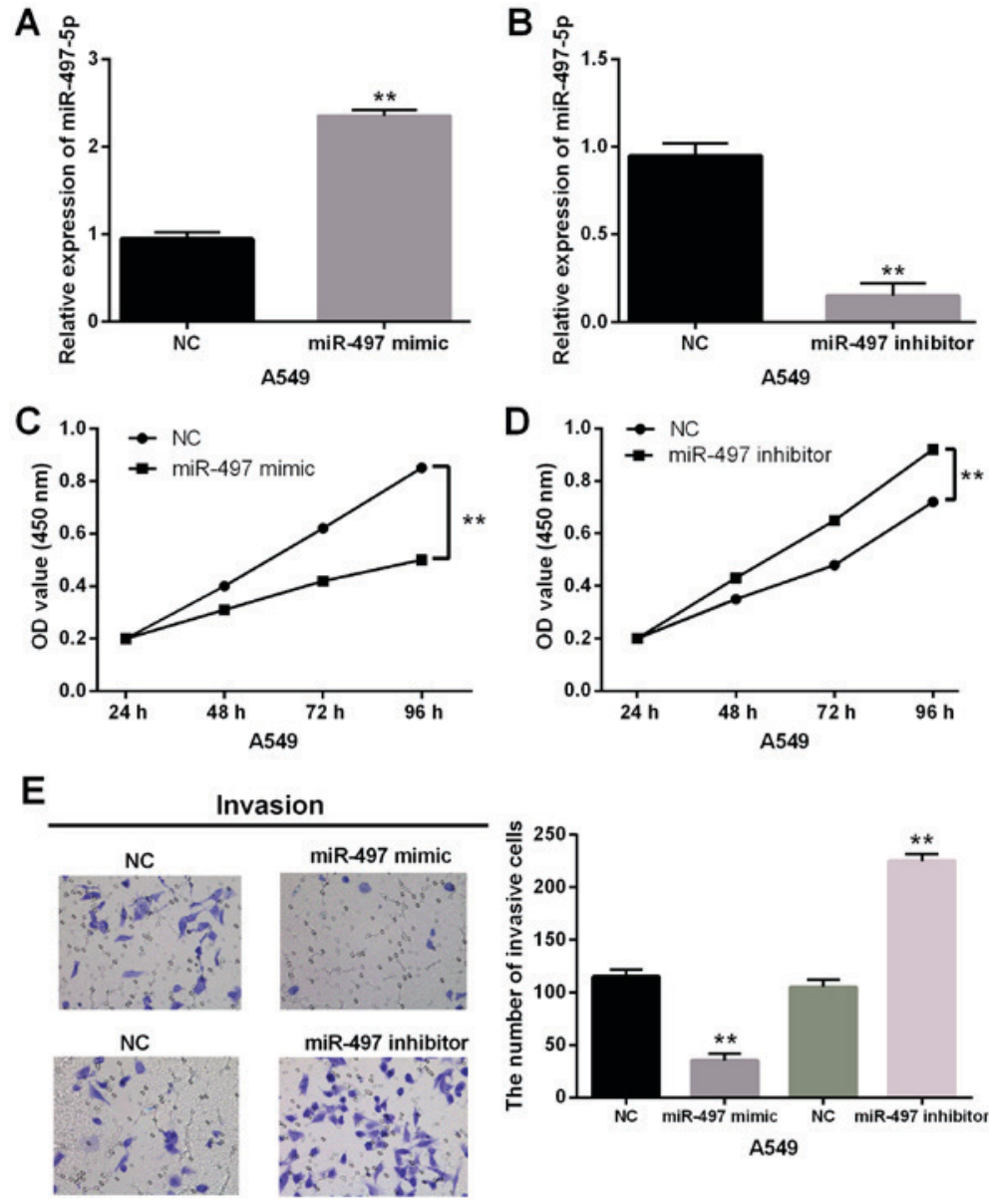

Figure 2. Overexpression of miR-497-5p inhibits cell proliferation and invasion in NSCLC. (A and B) The miR-497-5p expression was examined in A549 cells with miR-497-5p mimics or inhibitor via RT-qPCR. (C and D) Cell proliferation was measured in cells with miR-497-5p mimics or inhibitor. (E) Cell invasion analysis in A549 cells with miR-497-5p mimics or inhibitor was detected. ${ }^{* *} \mathrm{P}<0.01$.

\section{A $\quad$ MiR-497.5p}

Wild of FGF 23 '-UTR Mutant of FGF2 3 '-UTR

\section{B}

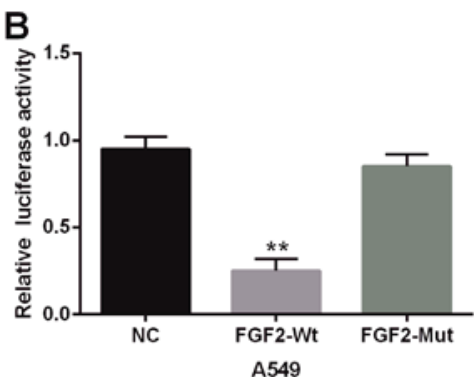

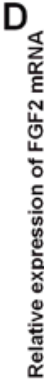

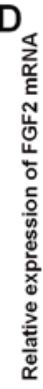

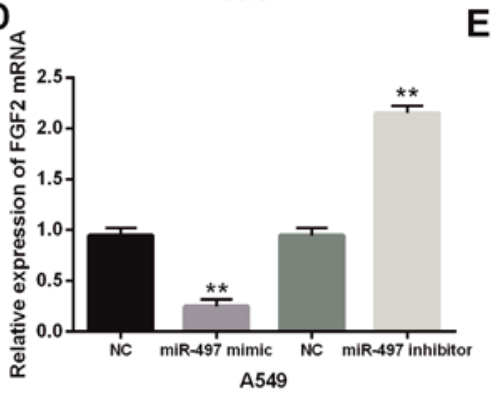

3'...UAAUU UAAAAU AU UU GCU GCUA....5'

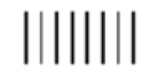

5'.........UGUUUGGUUCACACGACGAC....

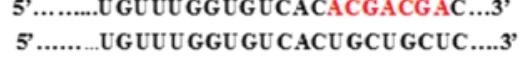

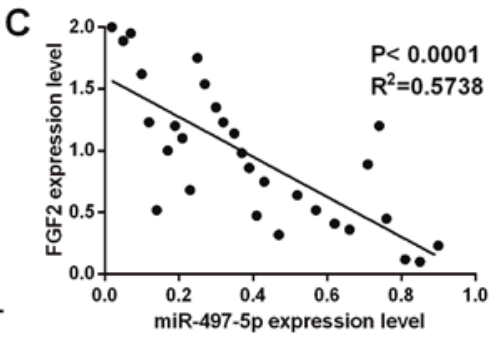

E

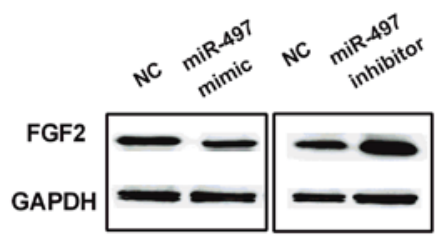

Figure 3. FGF2 is a downstream target of miR-497-5p in NSCLC. (A) The binding site of miR-497-5p on the 3'-UTR of FGF2. (B) Luciferase reporter assays (C) The correlation between miR-497-5p and FGF2. (D and E) The expression of FGF2 was analyzed in cells with miR-497-5p mimics or inhibitor. ${ }^{* *} \mathrm{P}<0.01$. 


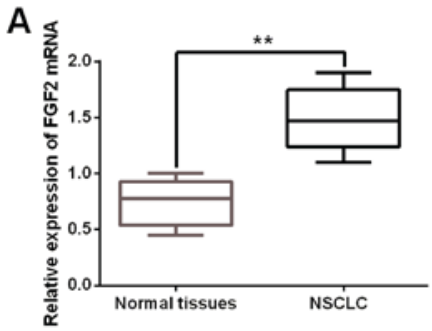

D

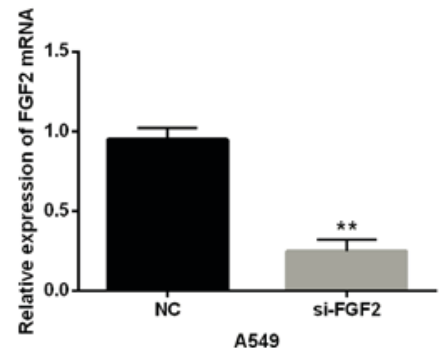

$\mathbf{F}$

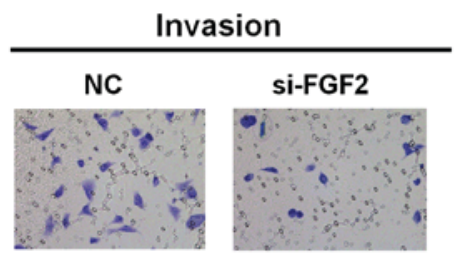

B

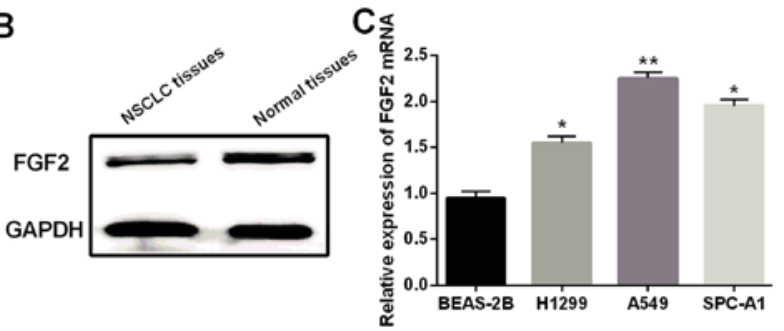

E
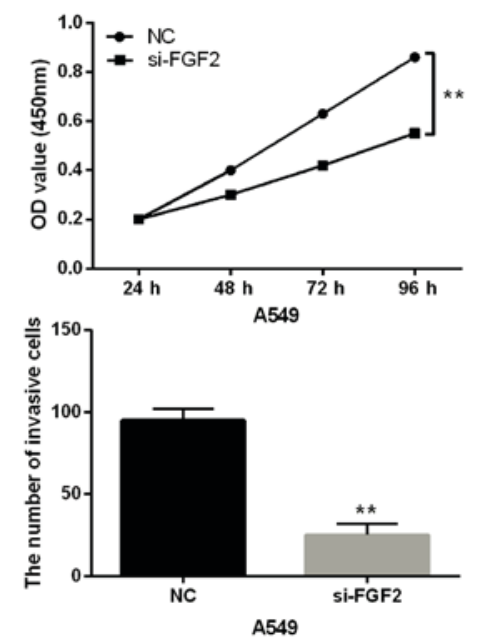

Figure 4. Upregulation of FGF2 promotes cell proliferation and invasion in NSCLC. (A and B) The mRNA and protein expression of FGF2 in NSCLC tissues was detected. (C) FGF2 expression in H1299, A549, SPC-A1 and BEAS-2B cell lines. (D) Expression of FGF2 was measured in cells with FGF2 siRNA. (E) Cell proliferation was measured in cells with FGF2 siRNA. (F) Cell invasion analysis in A549 cells with FGF2 siRNA was detected. ${ }^{*} \mathrm{P}<0.05,{ }^{* *} \mathrm{P}<0.01$.
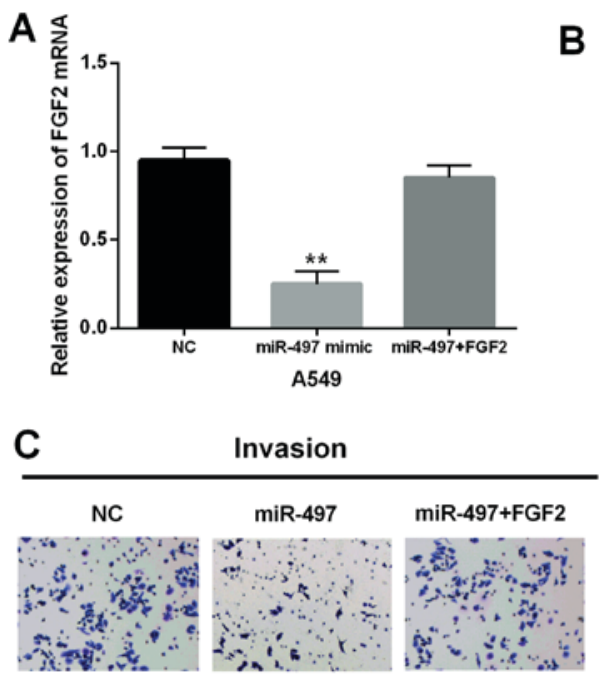

B
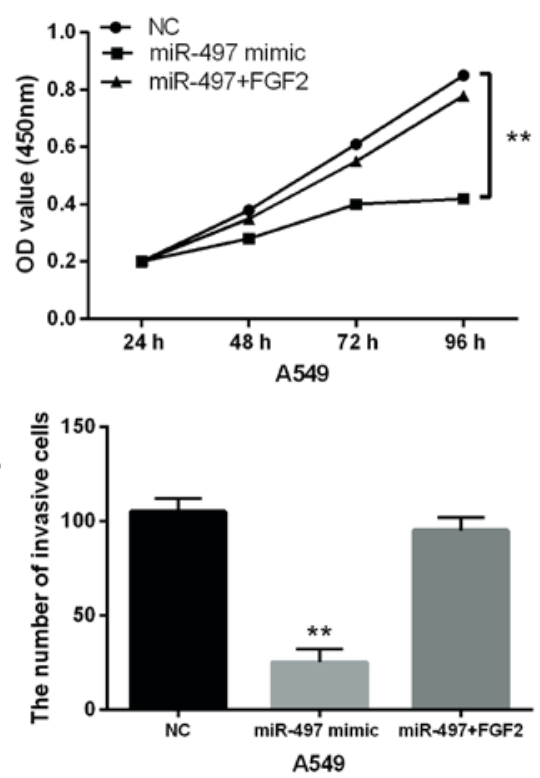

Figure 5. Overexpression of FGF2 impairs the suppressive effect of miR-497-5p in NSCLC. (A) The expression of FGF2 was measured in A549 cells with FGF2 vector and miR-497-5p mimics. (B) Cell proliferation was measured in A549 cells with FGF2 vector and miR-497-5p mimics. (C) Cell invasion in A549 cells with FGF2 vector and miR-497-5p mimics was measured. ${ }^{* *} \mathrm{P}<0.01$.

NSCLC tissues (Fig. 4A and B) and H1299, A549 and SPC-A1 cell lines (Fig. 4C). Moreover, high FGF2 expression was associated with TNM stage $(\mathrm{P}=0.009)$ and distant metastases $(\mathrm{P}=0.02$; Table II). Next, the effect of FGF2 was investigated in NSCLC by transfecting FGF2 siRNA in A549 cells. First, we found that FGF2 siRNA apparently reduced the expression of FGF2 (Fig. 4D). Moreover, silencing of FGF2 prevented proliferation of A549 cells (Fig. 4E). Similarly, knockdown of FGF2 also inhibited cell invasion in A549 cells (Fig. 4F). In conclusion, it was confirmed that FGF2 functions as an oncogene in NSCLC by promoting cell proliferation and invasion.

Upregulation of FGF2 impairs the inhibitory action of miR497-5p in NSCLC. Finally, the interaction between miR-497-5p 
Table II. Relationship between the FGF2 expression and the clinicopathological characteristics of NSCLC patients.

\begin{tabular}{|c|c|c|c|c|}
\hline \multirow[b]{2}{*}{ Characteristics } & \multirow{2}{*}{$\begin{array}{l}\text { No. of cases } \\
\qquad(n=108)\end{array}$} & \multicolumn{2}{|c|}{ FGF2 } & \multirow[b]{2}{*}{ P-value } \\
\hline & & High & Low & \\
\hline Age (years) & & & & 0.26 \\
\hline$\geq 60$ & 35 & 24 & 11 & \\
\hline$<60$ & 73 & 42 & 31 & \\
\hline Sex & & & & 0.08 \\
\hline Male & 42 & 23 & 19 & \\
\hline Female & 66 & 38 & 28 & \\
\hline Tumor size (cm) & & & & 0.63 \\
\hline$<5$ & 50 & 37 & 13 & \\
\hline$\geq 5$ & 58 & 38 & 20 & \\
\hline TNM stage & & & & $0.009^{\mathrm{a}}$ \\
\hline $\mathrm{I}+\mathrm{II}$ & 34 & 21 & 13 & \\
\hline III + IV & 74 & 45 & 29 & \\
\hline Distant metastases & & & & $0.02^{\mathrm{a}}$ \\
\hline Negative & 78 & 48 & 30 & \\
\hline Positive & 30 & 18 & 12 & \\
\hline Differentiation & & & & 0.053 \\
\hline $\mathrm{I}+\mathrm{II}$ & 61 & 35 & 26 & \\
\hline III + IV & 47 & 28 & 19 & \\
\hline
\end{tabular}

Statistical analyses were performed by the $\chi^{2}$ test. ${ }^{\text {a }}<<0.05$ was considered significant.

and FGF2 was explored through co-transfecting the miR497-5p mimics and the FGF2 vector into A549 cells. A decrease in FGF2 expression induced by the miR-497-5p mimics was found to be restored by the FGF2 vector (Fig. 5A). Functionally, the CCK-8 assay indicated that upregulation of FGF2 impaired the inhibitory effect of miR-497-5p on cell proliferation in NSCLC (Fig. 5B). Moreover, the inhibitory effect of miR-497-5p on cell invasion was also reversed by upregulation of FGF2 in A549 cells (Fig. 5C). In conclusion, overexpression of FGF2 impaired the suppressive effect of miR-497-5p in NSCLC.

\section{Discussion}

Various microRNAs (miRNAs) that can assess the risk of tumor progression and metastasis have been reported for use in non-small cell lung cancer (NSCLC) (22-24). In the present study, the expression of miR-497-5p was significantly reduced, and low expression of miR-497-5p was closely related to TNM stage and distant metastases of NSCLC. Similarly, miR-497 was reported to be downregulated and to inhibit tumor growth in NSCLC (25), which was consistent with our results. Besides, downregulation of miR-497 was also observed in osteosarcoma (26), thyroid (27) and colorectal cancer (28). These findings suggest that abnormal miR-497-5p expression is involved in the pathogenesis of NSCLC.

The effect of miR-497-5p on cell proliferation and invasion was also investigated in NSCLC. Li et al demonstrated that overexpression of miR-497 suppressed cell proliferation and invasion in human retinoblastoma (29). Similar inhibition of cell proliferation and invasion by miR-497-5p was also detected in NSCLC. Moreover, miR-497 has been found to regulate several target genes to mediate the development of human cancers. For example, miR-497 inhibited proliferation of hepatocellular carcinoma cells and induced cell apoptosis through targeting YAP1 (30). Ruan et al proposed that miR-497 inhibited proliferation, migration, and invasion of osteosarcoma cells via targeting AMOT (31). In the present study, FGF2 was shown to be a downstream and direct target of miR-497-5p in NSCLC.

Subsequently, the alteration of FGF2 expression and its effects were investigated, and the regulation mechanism of miR-497-5p in NSCLC was further elucidated. Upregulation of FGF2 was identified in NSCLC, which promoted proliferation and invasion of NSCLC cells. Similarly to our results, Cheng et al demonstrated that FGF2 expression was increased and knockdown of FGF2 inhibited proliferation and invasion of NSCLC cells (32). Besides, a negative correlation between miR-497-5p and FGF2 expression was revealed in NSCLC tissues. In addition, upregulation of FGF2 impaired the inhibitory effect of miR-497-5p in NSCLC. Similarly, previous studies have also shown that many miRNAs negatively regulated FGF2 expression, such as miR-195 (33), miR-205 (34) and miR-646 (35). Moreover, He et al implied that miR-16 targeting FGF2 inhibited proliferation and invasion of nasopharyngeal carcinoma cells (36). In the present study, miR-497-5p also inhibited proliferation and invasion of NSCLC cells by targeting FGF2.

In conclusion, in the present study, we revealed the inhibitory effect of miR-497-5p on the progression of NSCLC through targeting FGF2. This suggests that miR-497-5p acts as a novel tumor suppressor in NSCLC. In addition, FGF2 was a downstream target of miR-497-5p in NSCLC, indicating that FGF2 was involved in the regulatory mechanism of miR-497-5p. Further research is needed to investigate whether miR-497-5p is a useful biomarker and therapeutic target for NSCLC progression.

\section{Acknowledgements}

Not applicable.

\section{Funding}

Not applicable.

\section{Availability of data and materials}

The datasets used and/or analyzed during the present study are available from the corresponding author on reasonable request.

\section{Authors' contributions}

$\mathrm{XH}$ wrote the manuscript and contributed to the conception of the study. LW performed the data analysis. WL contributed to the data acquisition and analysis, and revised the manuscript. FL worked on aspects of the study relating to NSCLC patients. All authors read and approved the final manuscript. 


\section{Ethics approval and consent to participate}

The study was approved by the Institutional Ethics Committee of People's Hospital of Rizhao (Rizhao, China). Signed informed consents were obtained from the patients or the guardians.

\section{Patient consent for publication}

Not applicable.

\section{Competing interests}

The authors declare that they have no competing interests.

\section{References}

1. Chen W, Zheng R, Baade PD, Zhang S, Zeng H, Bray F, Jemal A, $\mathrm{Yu}$ XQ and He J: Cancer statistics in China, 2015. CA Cancer J Clin 66: 115-132, 2016

2. Laskin JJ and Sandler AB: State of the art in therapy for non-small cell lung cancer. Cancer Invest 23: 427-442, 2005.

3. Ma L, Qiu B, Zhang J, Li QW, Wang B, Zhang XH, Qiang MY, Chen ZL, Guo SP and Liu H: Survival and prognostic factors of non-small cell lung cancer patients with postoperative locoregional recurrence treated with radical radiotherapy. Chin J Cancer 36: 93, 2017.

4. Murray N: Reality check for pemetrexed and maintenance therapy in advanced non-small-cell lung cancer. J Clin Oncol 32: 482-483, 2014

5. Ferguson MK: Diagnosing and staging of non-small cell lung cancer. Hematol Oncol Clin North Am 4: 1053-1068, 1990.

6. Bartel DP: MicroRNAs: Genomics, biogenesis, mechanism, and function. Cell 116: 281-297, 2004.

7. Del Vescovo V, Grasso M, Barbareschi M and Denti MA: MicroRNAs as lung cancer biomarkers. World J Clin Oncol 5: 604-620, 2014.

8. Yao Y, Shen H, Zhou Y, Yang Z and Hu T: MicroRNA-215 suppresses the proliferation, migration and invasion of non-small cell lung carcinoma cells through the downregulation of matrix metalloproteinase-16 expression. Exp Ther Med 15: 3239-3246, 2018.

9. Wei L and Ran F: MicroRNA-20a promotes proliferation and invasion by directly targeting early growth response 2 in non-small cell lung carcinoma. Oncol Lett 15: 271-277, 2018.

10. Pang PC, Shi XY, Huang WL and Sun K: miR-497 as a potential serum biomarker for the diagnosis and prognosis of osteosarcoma. Eur Rev Med Pharmacol Sci 20: 3765-3769, 2016.

11. Pengcheng S, Ziqi W, Luyao Y, Xiangwei Z, Liang L, Yuwei L, Lechen L and Wanhai X: MicroRNA-497 suppresses renal cell carcinoma by targeting VEGFR-2 in ACHN cells. Biosci Rep 37: 37, 2017.

12. Wu R, Tang S, Wang M, Xu X, Yao C and Wang S: MicroRNA-497 induces apoptosis and suppresses proliferation via the $\mathrm{Bcl}-2 /$ Bax-caspase9-caspase3 pathway and cyclin D2 protein in HUVECs. PLoS One 11: e0167052, 2016.

13. Tao L, Zhang CY, Guo L, Li X, Han NN, Zhou Q and Liu ZL: MicroRNA-497 accelerates apoptosis while inhibiting proliferation, migration, and invasion through negative regulation of the MAPK/ERK signaling pathway via RAF-1. J Cell Physiol 233: 6578-6588, 2017.

14. Hu J, Xu JF and Ge WL: MiR-497 enhances metastasis of ora squamous cell carcinoma through SMAD7 suppression. Am J Transl Res 8: 3023-3031, 2016.

15. Song KH, Cho H, Kim S, Lee HJ, Oh SJ, Woo SR, Hong SO, Jang HS, Noh KH, Choi CH, et al: API5 confers cancer stem cell-like properties through the FGF2-NANOG axis. Oncogenesis 6: e285, 2017.

16. Khurana A, Liu P, Mellone P, Lorenzon L, Vincenzi B, Datta K, Yang B, Linhardt RJ, Lingle W, Chien J, et al: HSulf-1 modulates FGF2- and hypoxia-mediated migration and invasion of breast cancer cells. Cancer Res 71: 2152-2161, 2011.
17. Coleman SJ, Chioni AM, Ghallab M, Anderson RK, Lemoine NR Kocher HM and Grose RP: Nuclear translocation of FGFR1 and FGF2 in pancreatic stellate cells facilitates pancreatic cancer cell invasion. EMBO Mol Med 6: 467-481, 2014.

18. Joy A, Moffett J,Neary K, Mordechai E, Stachowiak EK, Coons S, Rankin-Shapiro J, Florkiewicz RZ and Stachowiak MK: Nuclear accumulation of FGF-2 is associated with proliferation of human astrocytes and glioma cells. Oncogene 14: 171-183, 1997.

19. Sooman L, Freyhult E, Jaiswal A, Navani S, Edqvist PH, Pontén F, Tchougounova E, Smits A, Elsir T, Gullbo J, et al: FGF2 as a potential prognostic biomarker for proneural glioma patients. Acta Oncol 54: 385-394, 2015.

20. Xue G, Yan HL, Zhang Y, Hao LQ, Zhu XT, Mei Q and Sun SH: c-Myc-mediated repression of miR-15-16 in hypoxia is induced by increased HIF-2 $\alpha$ and promotes tumor angiogenesis and metastasis by upregulating FGF2. Oncogene 34: 1393-1406, 2015.

21. Livak KJ and Schmittgen TD: Analysis of relative gene expression data using real-time quantitative PCR and the 2(-Delta Delta C(T)) method. Methods 25: 402-408, 2001.

22. Yang CL, Zheng XL, Ye K, Ge H, Sun YN, Lu YF and Fan QX: MicroRNA-183 acts as a tumor suppressor in human non-small cell lung cancer by down-regulating MTA1. Cell Physiol Biochem 46: 93-106, 2018.

23. Liu M, Zhang Y, Zhang J, Cai H, Zhang C, Yang Z, Niu Y, Wang $\mathrm{H}$, Wei X, Wang W, et al: MicroRNA-1253 suppresses cell proliferation and invasion of non-small-cell lung carcinoma by targeting WNT5A. Cell Death Dis 9: 189, 2018.

24. Wang J, Wang Y, Sun D, Bu J, Ren F, Liu B, Zhang S, Xu Z, Pang S and Xu S: miR-455-5p promotes cell growth and invasion by targeting SOCO3 in non-small cell lung cancer. Oncotarget 8 : 114956-114965, 2017.

25. Huang C, Ma R, Yue J, Li N, Li Z and Qi D: MiR-497 suppresses YAP1 and inhibits tumor growth in non-small cell lung cancer. Cell Physiol Biochem 37: 342-352, 2015

26. Sun Z, Li A, Yu Z, Li X, Guo X and Chen R: MicroRNA-497-5p suppresses tumor cell growth of osteosarcoma by targeting ADP ribosylation factor-like protein 2. Cancer Biother Radiopharm 32: 371-378, 2017.

27. Wang P, Meng X, Huang Y, Lv Z, Liu J, Wang G, Meng W, Xue S, Zhang Q, Zhang P, et al: MicroRNA-497 inhibits thyroid cancer tumor growth and invasion by suppressing BDNF. Oncotarget 8 : 2825-2834, 2017

28. Xu Y, Chen J, Gao C, Zhu D, Xu X, Wu C and Jiang J: MicroRNA-497 inhibits tumor growth through targeting insulin receptor substrate 1 in colorectal cancer. Oncol Lett 14: 6379-6386, 2017.

29. Li J, Zhang Y, Wang X and Zhao R: microRNA-497 overexpression decreases proliferation, migration and invasion of human retinoblastoma cells via targeting vascular endothelial growth factor A. Oncol Lett 13: 5021-5027, 2017.

30. Zhang L, Yu Z, Xian Y and Lin X: microRNA-497 inhibits cell proliferation and induces apoptosis by targeting YAP1 in human hepatocellular carcinoma. FEBS Open Bio 6: 155-164, 2016.

31. Ruan WD, Wang P, Feng S, Xue Y and Zhang B: MicroRNA-497 inhibits cell proliferation, migration, and invasion by targeting AMOT in human osteosarcoma cells. Onco 9Targets Ther 9: 303-313, 2016.

32. Cheng Z, Ma R, Tan W and Zhang L: MiR-152 suppresses the proliferation and invasion of NSCLC cells by inhibiting FGF2. Exp Mol Med 46: e112, 2014

33. Zhang X, Xu J, Jiang T, Liu G, Wang D and Lu Y: MicroRNA-195 suppresses colorectal cancer cells proliferation via targeting FGF2 and regulating Wnt/ $\beta$-catenin pathway. Am J Cancer Res 6: 2631-2640, 2016.

34. Hu Y, Qiu Y, Yagüe E, Ji W, Liu J and Zhang J: miRNA-205 targets VEGFA and FGF2 and regulates resistance to chemotherapeutics in breast cancer. Cell Death Dis 7: e2291, 2016.

35. Sun XH, Geng XL, Zhang J and Zhang C: miRNA-646 suppresses osteosarcoma cell metastasis by downregulating fibroblast growth factor 2 (FGF2). Tumour Biol 36: 2127-2134, 2015.

36. He Q, Ren X, Chen J, Li Y, Tang X, Wen X, Yang X, Zhang J, Wang Y, Ma J, et al: miR-16 targets fibroblast growth factor 2 to inhibit NPC cell proliferation and invasion via PI3K/AKT and MAPK signaling pathways. Oncotarget 7: 3047-3058, 2016.

This work is licensed under a Creative Commons Attribution-NonCommercial-NoDerivatives 4.0 International (CC BY-NC-ND 4.0) License. 